\title{
A Case Report of Twenty-Six Years Follow- up of Bronchio-Alveolar Lung Cancer Using Local Surgical and Repeated Radiation Techniques
}

\author{
Ralf Wilkowski $^{1}$, Alexander Muacevic ${ }^{1}$, Christoph Fürweger ${ }^{2}$ \\ 1. European CyberKnife Center Munich 2. Chief Medical Physicist, European CyberKnife Center Munich
}

$\square$ Corresponding author: Alexander Muacevic, alexander.muacevic@cureus.com

Disclosures can be found in Additional Information at the end of the article

\section{Abstract}

This case report describes a long follow-up of a patient treated for lung cancer by local therapy only using surgery and multiple radiation applications.

Categories: Radiation Oncology

Keywords: non-small cell lung cancers (nsclc), radiation therapy, linac, cyberknife, lung radiosurgery, hypofractionation

\section{Introduction}

The treatment of non-small cell lung cancer is defined in primarily localized and metastatic disease. Surgery, radiation therapy and chemotherapy are applied depending on the extent of the disease. Solitary metastasized situations with relatively long survival times have been described [1-2]. By definition, these cases are considered as a metastasized status but still may be regarded as a loco-regional tumor progression. Treatment decisions in these situations are always individual, dependent on the patients' preference, and require a great deal of experience in the use of either treatment method. In most cases, repeated surgery and/or chemotherapy are proposed. Recently, also repeated radiation treatments are considered if there is not too much of dose overlap and/or a small clinical target volume involved. Repeated radiation treatments in the same lung localization are not yet well-defined and biological tissue effects, such as late toxicity, are described [3-4]. We here present a rare case of a lung cancer patient who was treated over twenty-six years with surgery and multiple radiation treatments, mainly in the region of the right middle lobe.

\section{Case Presentation}

Review began 06/19/2012 Published 08/08/2012

C Copyright 2012

Wilkowski et al. This is an open access article distributed under the terms of the Creative Commons Attribution License CC-BY 3.0., which permits unrestricted use, distribution, and reproduction in any medium, provided the original author and source are credited.
We report on a 76-year-old female patient who was first seen in our institution in 2007 with a recurrent broncho-alveolar carcinoma treated initially in 1986 for a left-sided pT2, N0, M0, G2 tumor with lobectomy and atypical left lower lobe resection. A summary of therapeutic and diagnostic procedures is given in Table 1. 


\section{Cureus}

\begin{tabular}{|c|c|c|c|}
\hline Date & Clinical Status & Procedure & $\begin{array}{l}\text { Radiation Therapy: Dose, Target } \\
\text { vol. }\end{array}$ \\
\hline 1986 & $\begin{array}{l}\text { NSCLC - } \\
\text { pT2,pN0,M0,G2,R0 } \\
\text { Broncho-alveolar carcinoma }\end{array}$ & $\begin{array}{l}\text { Lobectomy, left lower lobe } \\
\text { resection }\end{array}$ & \\
\hline 09/2005 & $\begin{array}{l}\text { Recurrence right middle } \\
\text { lobe }\end{array}$ & PET/CT, biopsy & \\
\hline \multirow{3}{*}{$02 / 2007$} & Progression & & \\
\hline & $\begin{array}{l}\text { Recurrence right middle } \\
\text { lobe }\end{array}$ & Cyberknife & 1x24Gy, 13cc \\
\hline & Metastasis left upper lobe & Radiotherapy & $7 x 5 G y, 71 c c$ \\
\hline$>04 / 2008$ & Remission & CT & \\
\hline \multirow{3}{*}{ 08/2008 } & Recurrence / Progression & $\mathrm{PET} / \mathrm{CT}$ & \\
\hline & $\begin{array}{l}\text { Recurrence right middle } \\
\text { lobe }\end{array}$ & Radiotherapy & $8 x 5 G y, 46 c c$ \\
\hline & New Met. left upper lobe & Radiotherapy & $8 x 5 G y, 8 c c$ \\
\hline$>02 / 2009$ & Remission & $\mathrm{PET} / \mathrm{CT}$ & \\
\hline \multirow[b]{2}{*}{$11 / 2009$} & Recurrence & PET/CT & \\
\hline & $\begin{array}{l}\text { Recurrence right middle } \\
\text { lobe }\end{array}$ & Radiotherapy & $8 x 5 G y, 50 c c$ \\
\hline$>12 / 2010$ & Remission & CT & \\
\hline \multirow[b]{2}{*}{ 03/2011 } & Recurrence & Cytology & \\
\hline & $\begin{array}{l}\text { Right middle lobe / } \\
\text { bronchus }\end{array}$ & Radiotherapy & $6 x 8 G y, 46 c c$ \\
\hline >04/2012 & Partial remission & PET/CT & \\
\hline
\end{tabular}

\section{TABLE 1: Summary of therapeutic and diagnostic procedures}

Summary of therapeutic and diagnostic procedures over time, distinguished between right middle lobe [1] and the left upper lobe [2].

Until 2005, the patient was stable and then developed a suspected recurrent tumor formation in the right middle lobe (segment 5) and near the aortic arch in the left lung, diagnosed by PET/CT imaging. These lesions proved to be tumor tissue in the following bronchoscopic biopsy. No other tumors were found.

Because of the bilateral tumor expansion, the patient was not considered as a surgical 


\section{Cureus}

candidate. Chemotherapy was not applied due to the surprisingly long follow-up period without tumor progression. The patient was set on a wait-and-see strategy by the responsible oncologist. In the following CT investigation in 2007, there was documented progressive tumor growth of both lesions without evidence of any new tumor formations in the lung and systemically (Figure 1).
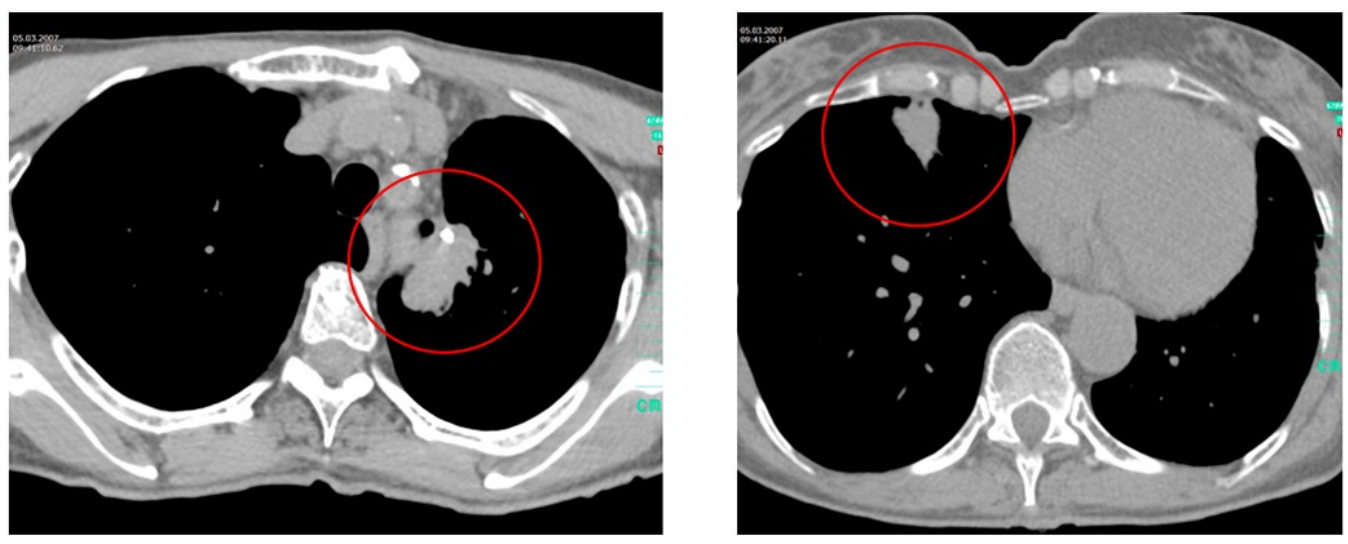

FIGURE 1: Left: CT March 07 before therapy of the left paraaortic tumor. Right: Right-sided lung metastasis, segment 5

Left: CT March 07 before therapy of the left para-aortic tumor. Right: Right-sided lung metastasis, segment 5

The interdisciplinary tumor board suggested performing a single session radiosurgery for the right-sided lung lesion and small field conventional radiation therapy for the left-sided paraaortal lesion. In March 2007, CyberKnife radiosurgery of the right-sided tumor was performed after CT-guided fiducial implantation with $24 \mathrm{~Gy}$ (75\% isodose) ray tracing dose calculation using a margin of $4 \mathrm{~mm}(\mathrm{CTV}, 13.2 \mathrm{cc})$. The recalculated dose with the later available Monte Carlo algorithm for inhomogeneity correction was 18 Gy to the $60 \%$ isodose. In addition, the paraaortal lesion was treated by hypofractionated three-dimensional conformal radiotherapy (five fractions, 7 x 5 Gy $70.5 \mathrm{cc}$ ) using a conventional $6 \mathrm{MeV}$ LINAC . After this therapy, the patient experienced slight pulmonary symptoms (CTC Grade 1). PET/CT was uneventful until August 2008 where a local recurrence was suspected in the right middle lobe (45.5 cc) and a new lesion in the left upper lobe. The para-aortic tumor was in complete remission. Because of the favorable follow-up, a new indication for hypofractionated radiation therapy was set and both lesions were treated with 8 x 5 Gy up to a total dose of 40 Gy using an eight field technique (conventional LINAC). The complete 2007 irradiated volume in the right middle lobe was integrated to the new dose plan. There were no new additional short-term side-effects. PET/CT imaging February 2009 showed a controlled tumor status without evidence of new tumor growth.

The next PET/CT control October 2009 showed again tumor activity in the right middle lobe and a new paracardial tumor formation. The tumor in the right middle lobe and the new lesion were again treated with 8 x 5 Gy (conventional LINAC). Fifteen cc of the right middle lobe had received by this time a cumulated dose of 1 x $24 \mathrm{~Gy}$ and two times 8 x $5 \mathrm{~Gy}$. Notwithstanding, the repeated treatment was well-tolerated by the patient, and she only suffered from a transient fatigue syndrome (CTC Grade 1) and mild coughing (CTC Grade 1).

In April 2011, the patient developed hemoptysis. The cytological evaluation showed malignant 


\section{Cureus}

cells of adenocarcinoma. Again, parts of the right-sided middle lobe were treated by hypofractionated radiotherapy with 6 × 8 Gy using an eight field technique (conventional LINAC). About $15 \mathrm{cc}$ of the anterior target volume had received up to now a cumulative dose of approximately $140 \mathrm{~Gy}$. Interestingly, again this therapy was associated with little adverse events (mild nausea).

The last follow-up control, as of April 2012, showed reduced tracer uptake at all treated areas of the lung without evidence of new tumor formations.
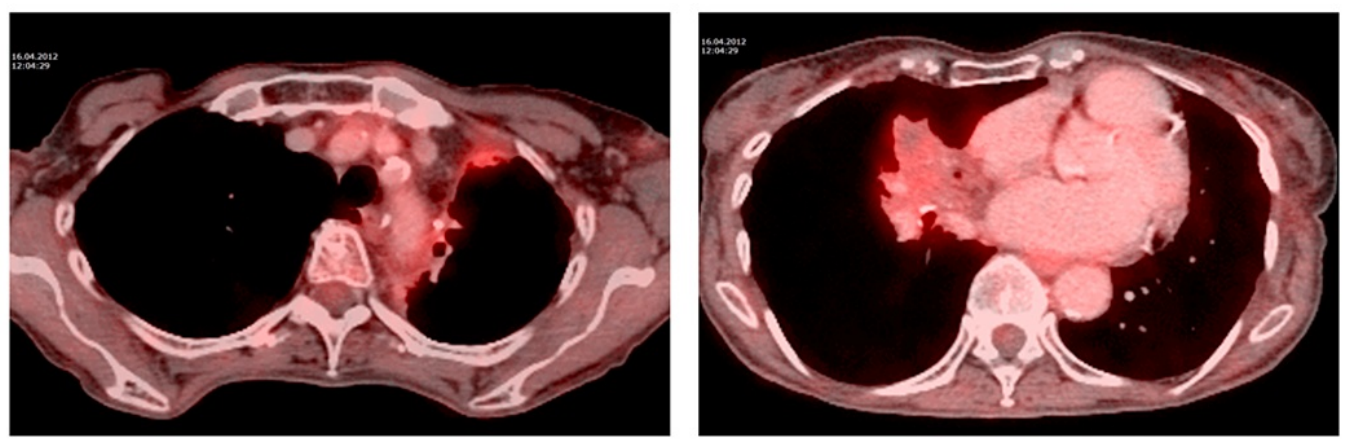

\section{FIGURE 2: Last PET-CT April 2012}

Complete remission of the left para-aortic tumor and reduced tracer enhancement in the right lung after multiple radiation treatments.

Complete remission of the left para-aortic tumor and reduced tracer enhancement in the right lung after multiple radiation treatments.

\section{Discussion}

The course of the disease of this 76-year-old woman and the treatment methods selected are very unusual and were based on individual treatment decisions by the responsible physicians and the patient, whose husband is a physician himself and was not in favor of any systemic chemotherapy regimes. Over 26 years, the bronchio-alveolar carcinoma was treated only with local therapy, initially surgery, and then repeated radiosurgery and hypofractionated radiation therapy. Segment five of the right lung (lower and middle lobe) was treated four times by radiotherapy with overlapping fields. A volume of $15 \mathrm{cc}$ received a cumulative dose of $140 \mathrm{~Gy}$, administered initially with SRS (CyberKnife) and then with SBRT regimens using hypofractionated radiotherapy. Between each course of treatment, a minimum time of 15 months elapsed. At no time was a Grade 3/4 toxicity observed.

Little information on re-irradiation is currently available in the literature.

Dworzecki, et al. [5] described just recently the feasibility of re-irradiation with cumulative doses above 110 Gy with acceptable toxicity using stereotactic radiotherapy in the lung. Serious side-effects with massive lung bleeding after re-irradiation of central lung tumors was described, mainly in large target volumes and central tumor locations, by Peulen, et al. [4]. In our case, temporary hemoptysis occurred in April 2011 which was most probably induced by local tumor recurrence (malignant cells found after cytologic evaluation) and not regarded as late radiation toxicity.

It may be speculated that the 15 month time intervals between each radiation treatment allowed tissue regeneration and accounted for the acceptable toxicity we found up to now. The 
real impact of time intervals needs to be studied in better established prospective protocols and cannot be answered in the framework of this case study. Tada, et al. [6] reported on the feasibility of repeated irradiations using longer time intervals.

Target volume definition becomes increasingly important with multiple radiation treatments [7]. We speculated that we defined too small a target volume in the right middle lobe, and therefore, local recurrences developed at the edge of the tumor repeatedly over time. On the other hand, it is well-known that increasing the target volume leads to increased risk of radiation-induced pneumonitis [3]. Therefore, a careful evaluation of the margins applied to the treatment volume has to be done case by case, taking into account individual patientrelated factors, such as size and location of the tumor, concomitant disease, local pretreatment, and systemic therapies.

\section{Conclusions}

The herein described case illustrated both the feasibility of multiple irradiations using SRS and hypofractionated radiotherapy and their effectiveness over time with acceptable treatment toxicity. Further studies are urgently needed to answer questions about the dose-volume relationship in terms of effectiveness and toxicity of multiple radiation exposures to the lung, and the influence of time intervals between radiation treatments.

\section{Additional Information \\ Disclosures}

Human subjects: Consent was obtained by all participants in this study. Conflicts of interest: In compliance with the ICMJE uniform disclosure form, all authors declare the following: Payment/services info: All authors have declared that no financial support was received from any organization for the submitted work. Financial relationships: Ralf Wilkowski declare(s) an alternate financial activity from Accuray, Inc. Ralf Wilkowski receives speaker honoraria from Accuray, Inc. Other relationships: All authors have declared that there are no other relationships or activities that could appear to have influenced the submitted work.

\section{References}

1. Hasselle MD, Haraf DJ, Rusthoven KE, Golden DW, Salgia R, Villaflor VM, Shah N, Hoffman PC, Chmura SJ, Connell PP, Vokes EE, Weichselbaum RR, Salama JK: Hypofractionated imageguided radiation therapy for patients with limited volume metastatic non-small cell lung cancer. J Thorac Oncol. 2012, 7:376-81. 10.1097/JTO.0b013e31824166a5

2. Mordant P, Arame A, De Dominicis F, Pricopi C, Foucault C, Dujon A, Le Pimpec-Barthes F, Riquet M: Which metastasis management allows long-term survival of synchronous solitary M1b non-small cell lung cancer?. Eur J Cardiothorac Surg. 2012, 41:617-622. 10.1093/ejcts/ezr042

3. Matsuo Y, Shibuya K, Nakamura M, Narabayashi M, Sakanaka K, Ueki N, Miyagi K, Norihisa Y, Mizowaki T, Nagata Y, Hiraoka M: Dose-Volume Metrics Associated With Radiation Pneumonitis After Stereotactic Body Radiation Therapy For Lung Cancer. Int J Radiat Oncol Biol Phys. 2012, 83:545-549. 10.1016/j.ijrobp.2012.01.018

4. Peulen H, Karlsson K, Lindberg K, Tullgren O, Baumann P, Lax I, Lewensohn R, Wersaell P: after reirradiation of pulmonary tumours with stereotactic body radiotherapy . Radiother Oncol. 2011, 101:260-266. 10.1016/j.radonc.2011.09.012

5. Dworzecki T, Idasiak A, Syguła D, Dworzecka U, Suwiński R: Stereotactic radiotherapy (SBRT) as a sole or salvage therapy in non-small cell lung cancer patients. Neoplasma. 2012, 59:114120.

6. Tada T, Fukuda H, Matsui K, Hirashima T, Hosono M, Takada Y, Inoue Y: Non-small-cell lung cancer: reirradiation for loco-regional relapse previously treated with radiation therapy. Int J Clin Oncol. 2005, 10:247-250. 


\section{Cureus}

7. Kramer GW, Gans S, Ullmann E, van Meerbeeck JP, Legrand CC, Leer JW: Hypofractionated external beam radiotherapy as retreatment for symptomatic non-small-cell lung carcinoma: an effective treatment?. Int J Radiat Oncol Biol Phys. 2004, 58:1388-1393. 